\title{
| COMMENTAIRE DE LA SOCIÉTÉ FRANÇAISE DE MÉTALLURGIE ET DES MATÉRIAUX
}

\author{
Henri-Paul Lieurade
}

\section{La métallurgie dans les industries mécaniques}

\section{Contexte}

Dans le secteur de la "Métallurgie et de la transformation des métaux", plus de 10000 entreprises sont concernées par le travail des métaux. Ce sont pour la plupart des PME/PMI qui peuvent être rassemblées en trois " métiers » génériques : la sous-traitance (usinage, emboutissage, forge, chaudronnerie, traitement de surface), la fabrication de composants et de petits équipements, la fabrication de machines et d'équipements industriels.

Ces entreprises mettent donc en forme et/ou en œuvre des matériaux qui sont majoritairement métalliques.

\section{2 | Les matériaux de la mécanique}

\subsection{Contexte}

Dans les applications industrielles visées, c'est le plus souvent la surface des composants structuraux qui est la plus sollicitée en service. En effet, les principaux types d'endommagement subis concernent la fatigue mécanique et la fatigue de contact, le frottement et l'usure, la corrosion et l'oxydation, qui tous se produisent essentiellement au voisinage de la surface.

C'est pourquoi, l'intérêt « métallurgique » de l'ingénieur mécanicien se porte le plus souvent sur le matériau de surface, le matériau substrat étant choisi, non seulement pour sa résistance mécanique mais surtout pour son faible coût, sa disponibilité et la constance de ses caractéristiques de base. 


\subsection{Les traitements de surface}

Si le nombre de nuances des "matériaux du mécanicien » est relativement limité, il n'en va pas de même pour les " matériaux de surface " générés par des traitements dont la variété est quasiment infinie. Ainsi dans ce domaine, I'industrie mécanique élabore et utilise les traitements superficiels de conversion (par action thermique, thermochimique ou mécanique), les traitement de conversion (anodisation, chromatation,...) et les dépôts par voie humide (revêtements chimiques, électrolytiques,...) ou par voie sèche (revêtements sous vide PVD ou CVD, projection thermique, ....).

Grâce aux possibilités apportées par ces traitements, on peut élaborer des surfaces fonctionnelles, qui répondent à une ou plusieurs fonctions (par exemple : résistance à l'usure + résistance à la corrosion + esthétique). La réalisation de co-dépôts, dépôts multi-couches ou composites permet ainsi de répondre à des sollicitations en service, diverses et combinées.

La notion d'intégrité des surfaces vient compléter ce panorama. Cette notion concerne la mise en forme des matériaux métalliques par les divers procédés de la mécanique, cités plus haut et qui créent le plus souvent des états superficiels complexes (et souvent néfastes) en terme d'écrovissage, contraintes résiduelles et rugosité. Il s'agit alors de maîtriser les conditions de mise en forme pour leur substituer des caractéristiques favorables de la surface (par exemple : optimisation de la microstructure de la peau de forgeage).

\subsection{Les verrous}

Les principaux verrous actuels dans l'élaboration de cette " métallurgie de la surface » sont les suivants :

- maîtrise des procédés de mise en œuvre, de façon à réduire la dispersion et à optimiser les performances des traitements;

- développement de moyens d'investigation, de mesure et de contrôle des couches traitées;

- prise en compte des règlements environnementaux (substitution de certains éléments chimiques : $\mathrm{CrVl}, \mathrm{Ni}, \ldots, \mathrm{REACH})$;

- simulation numérique des procédés. 


\section{Les assemblages}

\subsection{Contexte}

La tendance inéluctable dans la conception de composants et de structures " multi-matériaux ", plutôt que de pièces monolithiques, conduit à s'intéresser lourdement aux procédés d'assemblages de matériaux métalliques ou non. D'un point de vue "tenue en service", c'est alors au niveau de l'assemblage (par soudage, collage, boulonnage, rivetage...) que se situent généralement les endommagements, du fait des discontinuités géométriques, métallurgiques et physicochimiques, engendrées par le procédé mis en œuvre.

À titre d'exemple, on est capable aujourd'hui d'élaborer des aciers possédant une excellente soudabilité et une limite d'élasticité voisine de $1200 \mathrm{MPa}$. Pour ces nuances, un assemblage bout-à-bout réalisé par soudage à l'arc conserve les mêmes caractéristiques de résistance à la traction que celles du le métal de base, avec une zone de rupture située en dehors de la liaison soudée. En revanche, sollicité en fatigue, cet assemblage possède une limite de fatigue qui ne dépasse pas 100 MPa.

Dans ce domaine, les règles métier ont longtemps servi de vade mecum. Elles sont aujourd'hui insuffisantes car les matériaux et les procédés d'assemblage ont évolué et les applications industrielles sont de plus en plus complexes. Des travaux de recherche sont à entreprendre, d'une part pour préciser les mécanismes les plus souvent multi-physiques de l'opération de soudage, d'autre part pour apporter cette connaissance dans la modélisation numérique des phénomènes, permettant, à terme, de remplacer et de généraliser les règles métiers.

\subsection{Les verrous (cas des assemblages soudés)}

Parmi les nombreuses questions, certaines relèvent des mêmes démarches que celles déployées dans le cas des procédés d'élaboration des matériaux métalliques, on peut citer en particulier:

- la formation de textures pendant le soudage qui est particulièrement cruciale vis-à-vis de la prévision de la ténacité des liaisons et de l'application des contrôles non destructifs (détermination des constantes élastiques et de la propagation des ondes ultrasonores); en effet, les modèles de solidification connus sont insuffisants car ils ne tiennent pas compte de la taille de grains quand ceux-ci sont équiaxes; 
- les conditions d'apparition des défauts métallurgiques, lors de la solidification du bain fondu ;

- la présence de défauts en zone affectée thermiquement, tels que les phénomènes de liquation rencontrés en soudage multi-passes et de fissuration à mi-chaud;

- la prévision de la pénétration des cordons de soudure;

- l'estimation des champs de contraintes résiduelles, des distorsions et de la géométrie du cordon.

La réponse à ces questions se situe dans le cadre du développement d'une modélisation numérique, complète et donc multi-physique du procédé. Cette modélisation qui doit tenir compte, à la fois des phénomènes de transfert dans l'arc, de la formation du cordon de soudure et des transferts thermiques, doit conduire à une estimation prédictive du gradient de microstructures et des champs de contraintes et de déformations induits par le procédé considéré.

\section{4 | Le couple matériau/procédé}

\subsection{Contexte}

Comme cela a été noté plus haut, les industries mécaniques se préoccupent de la modélisation numérique des procédés de mise en forme et de mise en œuvre des matériaux métalliques. Dans chaque cas, le Cetim s'est associé à des équipes universitaires, des entreprises concernées, y compris des PME/PMI, et des centres techniques; on peut citer les cas suivants :

- le forgeage : le projet Simulforge a regroupé pendant 9 ans un consortium de laboratoires et d'entreprises qui a conduit à la fourniture du logiciel Forge3;

- le soudage : sur la base du logiciel Sysweld, le projet Musica s'est intéressé au développement d'un logiciel permettant de prévoir les contraintes et les déformations dans des structures soudées de grandes dimensions;

- l'emboutissage : un outil métier a été développé en associant des logiciels existants et l'expertise des entreprises de la mécanique et de la sidérurgie ;

- les assemblages mécaniques : le projet en cours, Mona Lisa, s'intéresse à la conception prédictive d'assemblages par rivetage, clinchage, vissage et sertissage. 


\subsection{Les verrous à lever}

Si les travaux recherche cités précédemment montrent l'intérêt porté à la simulation numérique des procédés, la pertinence industrielle de leurs livrables s'évalue, en particulier, à l'aune du niveau de connaissance des mécanismes physiques induits par chaque couple matériau/procédé. Les connaissances actuelles sont donc à compléter afin d'améliorer la prédictivité des résultats.

Par ailleurs, la validation des modèles numériques nécessite la mise en place d'une instrumentation et de bancs d'essais adaptés ; leur exploitation industrielle requiert des bases de données importantes et souvent spécifiques dont il convient de disposer.

Enfin, la modélisation numérique des procédés de fabrication $n$ 'intervient, actuellement, dans la boucle de conception, qu'une fois le composant défini géométriquement (par exemple par modèle $\mathrm{CAO}$ ). Un couplage plus précoce permettrait de mieux tirer parti de la simulation, en jouant sur la configuration du produit.

\section{5 | Évaluation du dommage}

\subsection{Contexte}

La notion de dommage peut être sujette à controverses, compte tenu de l'échelle à laquelle elle est définie. D'un point de vue industriel, cette notion renvoie à des questions bien concrètes telles que celles-ci :

- cette structure est-elle apte à assurer la fonction pour laquelle elle a été conçue?

- quelle durée de vie lui reste-t-il avant d'être considérée comme inapte à assurer sa fonction?

- peut-on prolonger sa durée de vie au-delà de la période prévue au cahier des charges?

Bien que basiques, les questions précédentes appellent des réponses souvent complexes qui dépendent, à la fois de l'état microstructural actuel du matériau (induit par l'endommagement en service), du contexte d'utilisation du composant considéré (conditions d'exploitation), des moyens de contrôle actuels et des moyens d'auscultation ou de suivi (monitoring) prévus. 


\subsection{La mesure du dommage}

La mesure de l'endommagement des structures métalliques est largement dépendante des méthodes et des moyens d'investigation employés. Elle ne doit cependant pas occulter une étude fine des mécanismes microstructuraux induits par le dommage, de façon à mettre en évidence les phénomènes précurseurs qui permettront, à la fois de choisir les techniques de mesures et d'étalonner celles-ci.

Les phénomènes de fatigue, de fatigue-fluage, de corrosion sous contrainte ou de vieillissement par irradiation sont concernés par de telles approches que rend possible l'évolution actuelle des techniques de microscopie et d'analyses radio-cristallographiques. Ces approches, en prenant en compte les aspects multi-échelles et en s'appuyant sur la modélisation numérique des mécanismes, doivent permettre de passer des modèles phénoménologiques à des approches physiques et prédictives. 